\title{
13 A genetic way to conic sections and planetary motion with Cabri-Géomètre
}

\author{
Herbert Möller \\ University of Münster \\ Germany
}

\begin{abstract}
There is a need to diminish the discrepancy between the high quality of tools and programs offered by information technology and the orientation of mathematics curricula to general abstract concepts. An example here is how the power of Cabri-Gémètre can be used to support students in their investigation and discovery of the most important known and two unknown properties of non degenerate conic sections (conics) which finally lead to an adequate representation of planetary orbits.
\end{abstract}

\section{Keywords}

Learning models, creativity, geometry.

\section{Introduction}

The growing quality of tools like the hand-held computer TI-92, and of programs like DERIVE and Cabri-Géomètre, does not automatically enhance the situation in school mathematics. Science-oriented curricula and the 'sociological law of inertia' are well-known obstacles. Therefore the development of suitable conceptions and the construction of teaching material using information technology are the most challenging tasks in the near future.

Our work is based on two conceptions called 'Spatial Algebra' and 'Elementary Analysis'. The didactical framework following the model of 'guided discovery learning' and some technical references are described in Möller (1995) and a typical treatment of conics and planetary motion before the advent of information technology is given in Castelnuovo et al. (1979).

\section{Well-known properties of conics}

In this first phase Cabri-Géomètre is used for static drawings. We start with a table-tennis ball lying on a sheet of paper and a torchlight which gives a cone of light turned on the ball. Examining the frontiers of the shadows on the paper and on the ball, the focal properties of the central conics and the representation of all 
non degenerate conics with the help of a directrix are discovered in the following way:

First of all, special cases of both properties can be seen in the sectional drawing perpendicular to the paper plane and containing the midpoint of the ball. Since the focal property is obtained through segments on the generators of the cone it is important that the second sphere of contact can be discovered with a 'shirt-shaped' figure. The eccentricity property of the directrix is easily found by projecting the points of the conic to the plane containing the corresponding circle of contact.

The general cases of both properties can be visualised with perspectives using the powerful construction tools of Cabri-Géomètre.

\section{Discovery of new tangent properties}

This second part is dominated by dynamic applications of Cabri-Géomètre. Since the representation of the planetary orbits is derived from Newton's laws of motion and of gravitation we need tangent properties to handle the velocity vectors. Two new results enable a completely 'genetic' treatment.

We start with the hint that there is a simple connection between a fixed directrix and each tangent of a given conic. Experimenting with Cabri-Géomètre it is easy to conjecture that the mapping in question is a rotation-stretching round

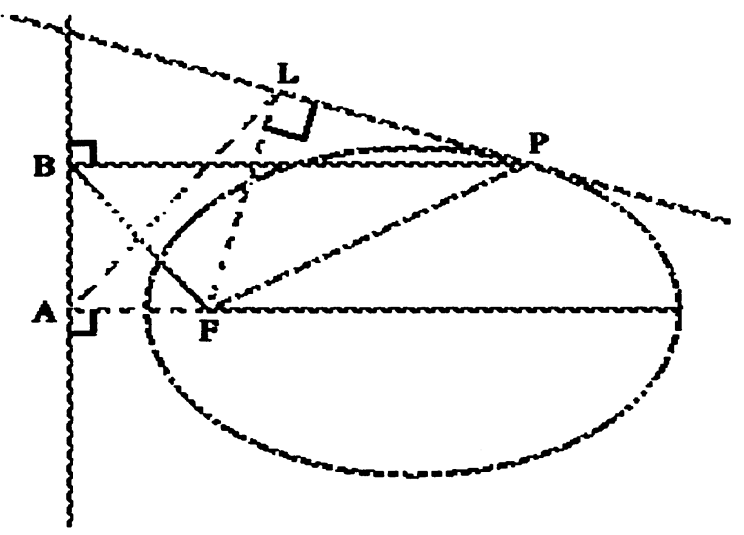
the corresponding focus for which the counter image of the tangent point of contact is the foot of the perpendicular from this point to the directrix (see fig. 1). The simple indirect proof uses the eccentricity property of the directrix and the triangular inequality.

Figure 1 
Velocity vectors are tangent vectors of the orbit. Therefore we search for a relation between tangent vectors and the corresponding radius vector of a conic in the vector space which has a focus as origin and which is spanned by three orthogonal unit vectors, the first of it $\left(\mathbf{e}_{1}\right)$ directed towards the nearest vertex of the conic and the third of it perpendicular to the plane of the section.

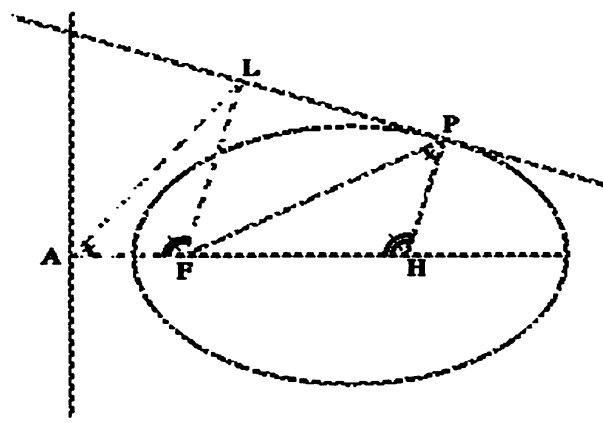

Figure 2

Since a rotation-stretching is an angle-preserving mapping, the foot $\mathrm{A}$ of the perpendicular from the focus $F$ to the directrix is mapped on the foot $L$ of the perpendicular to the tangent (figure 1). Therefore the triangles FBP and FAL are similar. Then, in figure 2 , the triangle HPF with HP parallel to FL is orientation-reversing, similar to FAL.

Now calculating the length of FH in terms of the magnitude $\mathbf{r}$ of $\mathbf{r}$ and of the eccentricity $\varepsilon$ we find that

$$
\mathbf{n}=r^{-1} \mathbf{r}+\varepsilon \cdot \mathbf{e}_{1}
$$

is a normal vector belonging to $\mathbf{r}$. Varying $\mathbf{r}$ and multiplying (1) by a positive constant $c$ we obtain the equation

$$
c . r^{-1} \mathbf{r}=c . \mathbf{n}-c . \varepsilon . \mathbf{e}_{1}
$$

which describes a circle with the radius $c$ where $c . n$ is always a normal vector belonging to $\mathbf{r}$ (see figure 3 ). Therefore we call (2) a 'track circle' representation of a conic. This equation will enable the genetic representation of the planetary orbits in the next section.

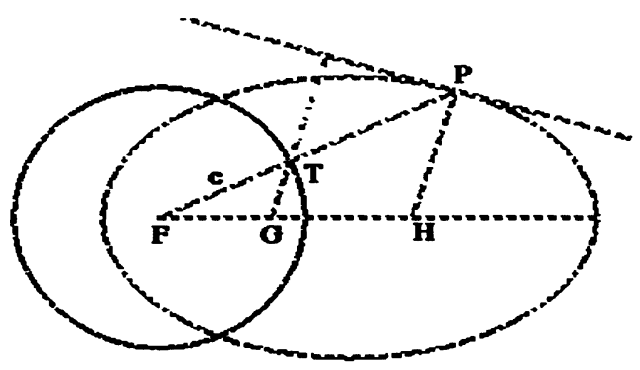

Figure 3

With Cabri-Géomètre using the track circle representation we let the students discover that the foot of the perpendicular from a focus to a tangent lies an the smallest circle through the vertices in the case of a central conic respectively on the tangent at the vertex when the conic is a parabola. This property prepares the discovery of the above-mentioned representation of planetary orbits. 
110 Information and communications technologies in school mathematics

Simulation of planetary motion

From Newton's laws we get the differential equation

$$
\mathbf{r}^{\prime \prime}=-g \cdot M \cdot r^{-3} \mathbf{r}
$$

where $\mathrm{g}$ is the gravitational constant and $M$ is the mass of the sun. Using CabriGémètre to simulate motions that have become discrete in a central force field on screen we find successive triangles with equal area. Since the magnitude of the vector product $\mathbf{r} \wedge \mathbf{r}^{\prime}$ equals the double of the area of the triangle generated by $\mathbf{r}$ and $\mathbf{r}^{\prime}$ it seems reasonable to consider the derivative of this vector product. We find $\left(\mathbf{r} \wedge \mathbf{r}^{\prime}\right)=\mathbf{r} \wedge \mathbf{r}^{\prime \prime}=\mathbf{0}$. With the theorem of constant functions which can be derived without integration and even without limits in elementary analysis we get that $\mathbf{d}=\mathbf{r} \wedge \mathbf{r}^{\prime}$ is a constant vector.

The inverse tool of Cabri-Géomètre enables the impressive simulation of motions becoming discrete in gravitational fields on screen. Motivated by the equality of triangular areas obtained by

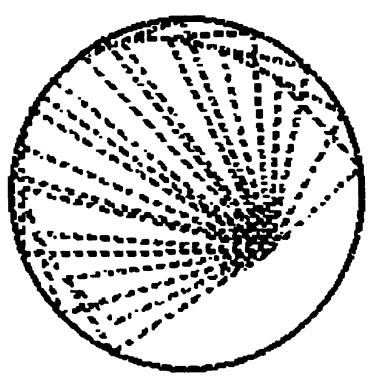

Figure 4 the first simulation we also construct the foot of the perpendicular to each difference vector (figure 4). The conjecture that these feet lie on a circle, is only approximately correct. Nevertheless, it leads to another conjecture connected with the track circle representation, namely that $\left(\mathbf{d} \wedge \mathbf{r}^{\prime}\right)^{\prime}$ is a constant multiple of $\left(r^{-1} \mathbf{r}\right)^{\prime}$ where $\mathbf{d} \wedge \mathbf{r}^{\prime}$ is a normal vector belonging to $r$. With simple calculations we get

$$
\left(g . M . r^{-1} \mathbf{r}+\mathrm{d} \wedge \mathbf{r}^{\prime}\right)^{\prime}=0 .
$$

Therefore the theorem of constant functions gives the track circle representation of a conic which in the case of planetary orbits must be an ellipse.

\section{References}

Castelnuovo, E., Gori-Giorgi, C. and Gori-Giorgi, D. (1979). Coniques et Gravitation Universelle. Educational Studies in Mathematics 10, 323-359.

Moller, H. (1995). How to develop 'discoveries'. In D. Watson and D. Tinsley (eds.) Integrating Information Technology into Education. Proceedings of the IFIP Working Conference in Barcelona. London: Chapman \& Hall. 